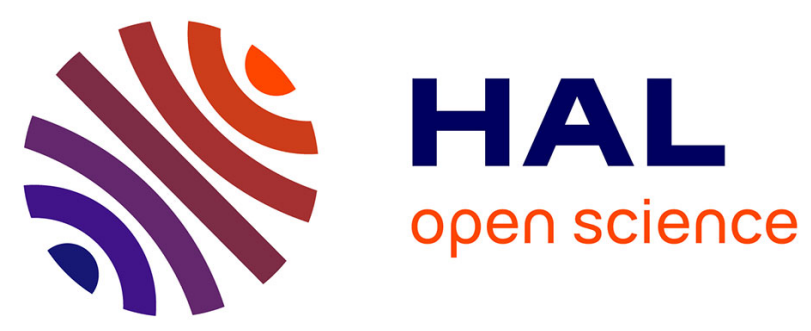

\title{
Pertinence cognitive des unités sémiotiques temporelles
}

Aline Frey, Alice Daquet, Sébastien Poitrenaud, Charles Tijus, Marcel Fremiot, Marcel Formosa, Lucie Prod'Homme, Jacques Mandelbrojt, Martine Timsit-Berthier, Philippe Bootz, et al.

\section{- To cite this version:}

Aline Frey, Alice Daquet, Sébastien Poitrenaud, Charles Tijus, Marcel Fremiot, et al.. Pertinence cognitive des unités sémiotiques temporelles. Musicae Scientiae, 2009, 13 (2), pp.415-440. 10.1177/102986490901300209 . hal-02430636

\section{HAL Id: hal-02430636 https://hal.science/hal-02430636}

Submitted on 10 Jan 2020

HAL is a multi-disciplinary open access archive for the deposit and dissemination of scientific research documents, whether they are published or not. The documents may come from teaching and research institutions in France or abroad, or from public or private research centers.
L'archive ouverte pluridisciplinaire HAL, est destinée au dépôt et à la diffusion de documents scientifiques de niveau recherche, publiés ou non, émanant des établissements d'enseignement et de recherche français ou étrangers, des laboratoires publics ou privés. 


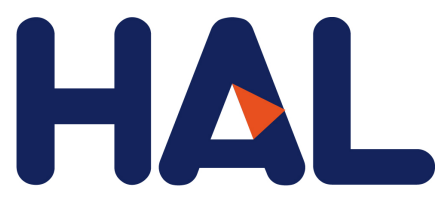

archives-ouvertes

\section{Pertinence cognitive des unités sémiotiques temporelles}

Aline Frey, Alice Daquet, Sébastien Poitrenaud, Charles Tijus, Marcel Fremiot, Marcel Formosa, Lucie Prod\&apos;homme, Jacques Mandelbrojt, Martine Timsit-Berthier, Philippe Bootz, et al.

\section{- To cite this version:}

Aline Frey, Alice Daquet, Sébastien Poitrenaud, Charles Tijus, Marcel Fremiot, et al.. Pertinence cognitive des unités sémiotiques temporelles. Musicae Scientiae, SAGE Publications, 2009, 13 (2), pp.415-440. 10.1177/102986490901300209 . hal-02430636

\section{HAL Id: hal-02430636 \\ https://hal.archives-ouvertes.fr/hal-02430636}

Submitted on 10 Jan 2020

HAL is a multi-disciplinary open access archive for the deposit and dissemination of scientific research documents, whether they are published or not. The documents may come from teaching and research institutions in France or abroad, or from public or private research centers.
L'archive ouverte pluridisciplinaire HAL, est destinée au dépôt et à la diffusion de documents scientifiques de niveau recherche, publiés ou non, émanant des établissements d'enseignement et de recherche français ou étrangers, des laboratoires publics ou privés. 


\title{
Pertinence cognitive des unités sémiotiques temporelles
}

\author{
ALINE FREY*, ALICE DAQUET*, SEBASTIEN POITRENAUD*, \\ CHARLES TIJUS*, MARCEL FREMIOT**, MARCEL FORMOSA**, \\ LUCIE PROD'HOMME**, JACQUES MANDELBROJT**, \\ MARTINE TIMSIT-BERTHIER**, PHILIPPE BOOTZ***, \\ XAVIER HAUTBOIS****, MIREILLE BESSON***** \\ * Laboratoire Cognitions Humaine \& Artificielle, CHArt, Université Paris 8 \\ ** Laboratoire Musique et Informatique de Marseille, Cité de la musique de Marseille \\ *** Laboratoire Paragraphe, Université Paris 8 \\ **** Institut d'Esthétique des Arts et Technologies, UMR 8153, \\ CNRS-Université Paris 1 \\ Institut de Neurosciences Cognitives de la Méditerranée, UMR 6193, \\ CNRS-Université de la Méditerranée
}

\begin{abstract}
- RÉSUMÉ
DandalignéedestravauxdePierreSchaeffer,daboratoireMusique et Informatique de Marseille(MIM)amisenévidencedesfigurestemporellessonoresdénommées Unités Sémiotiques Temporelles(UST)quicorrespondentàdessegmentsmusicaux quipossèdentper se,unesignificationtemporelleenraisondeleurorganisation morphologiqueetdynamique(Delalande,1996).

Danscetarticle,nousrapportonsladescriptiondesUSTetlesrésultatsdedeux expériences,menéesauprèsdemusiciensetdenormusiciensenutilisantunetâche decatégorisationlibrepourévaluerlapertinencedeSUST, commeunitéscognitives del'écoute duflux sonore. Les résultats montrentquelessegments musicaux relevantd'unemêmeUSTsontregroupésdefaçonsignificativeetqu'iln'yapas dedifférencesentremusiciensetnonmusiciensdansl'appréhensiondesUST.Ces résultatssontdiscutésauregarddelaformalisationdesUST.
\end{abstract}

\section{INTRODUCTION}

Les figures temporelles de Schering (1936), les groupements locaux de Reti (1951), les patterns familiers de Cooke (1959), l'idée de succession de segments indépendants, animés d'un mouvement intrinsèque, avec des zones de stabilité et d'instabilité de Meyer (1953, 1973) et de Kramer (2004), les vecteurs dynamiques de Francès (1958) et Imberty (1991) et en informatique musicale les patterns locaux de Ruwet 
(1987) et de Höppner (2003) et les Potential Actions du projet MusES (Pachet, Ramalho \& Carrive, 1996) sont autant de propositions d'existence de figures musicales temporelles.

Dans son traité de composition musicale, Reicha (1824, p. 329) supposait d'ailleurs déjà que la musique pourrait bien émettre des dessins géométriques comparables aux représentations visuelles. En effet, la musique, comme art du temps (Francès, 1958) ne dispose pas de figures singulières reconnues, qui seraient équivalant aux figures géométriques planes des arts de l'espace (triangle, rectangle, etc.), qui sont probablement universelles (Dehaene, Izard, Pica \& Spelke, 2006). De ce point de vue, la proposition d'Unités Sémiotiques Temporelles (UST) constitue, dans la continuité des travaux de Schaeffer (1966), la tentative d'une définition cohérente d'un petit nombre de figures temporelles (Delalande, Formosa, Frémiot, Gobin, Malbosc, Mandelbrojt, \& Pedler, 1996).

Les UST découlent de plus de 16 ans d'analyse du matériau musical. Depuis 1991, cette analyse est menée par les compositeurs et chercheurs du Laboratoire Musique et Informatique de Marseille (MIM) dans la continuité des travaux de P. Schaeffer qui définit, dans son "Traité des Objets musicaux ", le concept "d'objet sonore", reposant sur une "écoute réduite", comme "tout phénomène ou événement sonore perçu comme un ensemble, comme un tout cohérent et entendu dans une écoute réduite qui le vise pour lui-même, indépendamment de sa provenance et de sa signification" (Chion, 1983, p. 34).

Pour constituer des figures sonométriques, comme l'a préconisé Schaeffer, les chercheurs du MIM ont analysé collectivement un grand nombre de musiques classiques et contemporaines à partir d'une écoute orientée, en ayant comme descripteurs les caractères morphologiques et cinétiques du son. Leur écoute ne cherchait ni à entendre les successions de hauteur, ni les cadences, ni les nuances mais à repérer, puis à isoler les figures sonores en termes de vitesse, régulière ou non, de matière sonore, évoluant ou non, de trajectoire, brève ou longue. Cette analyse a conduit à un répertoire de 19 figures temporelles sonores, par ordre alphabétique: Chute, Contracté-Étendu, Élan, En flottement, En suspension, Étirement, Freinage, Lourdeur, Obsessionnel, Par vagues, Qui avance, Qui tourne, Qui veut démarrer, Sans direction par divergence d'information, Sans direction par excès d'information, Stationnaire, Sur l'erre, Suspension-Interrogation, Trajectoire inexorable. On trouvera de nombreux exemples sonores et l'analyse d'œuvres musicales dans le CD-Rom consacré aux UST (Favory, Formosa, Frémiot, Gobin, Malbosc, Mandelbrojt \& Prod'homme, 2002).

Pour les chercheurs du MIM, les Unités Sémiotiques Temporelles (UST) sont des catégories de segments musicaux qui possèdent, hors contexte, une signification temporelle en raison de leur organisation morphologique et dynamique (Delalande, 1996). «Unités» car ce sont des fragments musicaux, « Sémiotiques» car ces fragments semblent intrinsèquement porteurs d'un sens, et "Temporelles» car ce sens est fonction de la façon dont la matière sonore s'organise en évoluant dans le temps (Fremiot, 1999, Favory, 2007), la dimension temporelle étant une donnée constituante du son 
lui-même, comme le formule O. Messiaen (2000): «la musique ne se déroule pas dans un temps préalable, dans un temps physique, c'est elle qui engendre son propre temps".

Bien que l'utilité des UST ne soit plus à démontrer, puisqu'elles sont utilisées comme outil d'analyse et de composition (Frémiot \& Formosa, 2005) avec de nombreuses applications pédagogiques (Prod'homme, 2005), on ne connaît pas leur pertinence cognitive, c'est-à-dire leur bien-fondé comme unité de traitement du flux musical. Il se pourrait que ce soit une manière experte d'appréhender le flux musical en apprenant par un procédé comment coder le flux musical pour le segmenter en en catégorisant des extraits. Sans que ce soit un procédé, il se pourrait que l'expertise musicale soit nécessaire pour appréhender les UST. D'un autre côté, il se pourrait que ce soit une capacité commune que de traiter le flux musical en UST. Les UST, comme les figures géométriques, sont des catégories et relèvent de la recherche sur la catégorisation. Une catégorie permet de regrouper un ensemble d'objets ou des évènements non identiques en les considérant comme équivalents (Rosch \& Mervis, 1975). Il s'agit d'une entité cognitive à laquelle correspondent des mécanismes, des processus, des opérations et des actions qui sont dénommés sous le terme générique de « catégorisation " (Tijus \& Cordier, 2003). La catégorisation est l'un des mécanismes fondamentaux de la cognition humaine (Rosch, 1973). Elle permet d'organiser et de réduire la complexité de l'environnement, en le découpant et en le regroupant en objets qu'il attribue à différentes catégories (Tijus, 2004). Bien que le processus de constitution des catégories soit débattu (Poitrenaud, Richard \& Tijus, 2005), il est reconnu que percevoir, comprendre, raisonner, agir, communiquer, apprendre sont autant d'activités qui nécessitent de la catégorisation.

À cet égard, la catégorisation est un puissant indicateur de l'expertise qui s'acquiert progressivement après de nombreuses années d'études et de pratique d'un domaine particulier, qu'il s'agisse de vins (Solomon, 1990) ou de problèmes de physique (Chi, Feltovich \& Glaser, 1981). À l'expertise dans un domaine correspond une production de catégories et de descriptions plus fines, répétables, et basées sur des propriétés de structure et des propriétés fonctionnelles tandis que le manque d'expertise fait produire des catégories basées sur les propriétés de surface.

D'un autre côté, le savoir commun, implicite, non spécifique à un domaine en particulier peut relever de catégories acquises par l'expérience de chacun, comme cela a pu être montré pour les figures géométriques (Dehaene et al., 2006). En effet, dans le domaine spatial, on peut se demander en quoi des années de pratique du dessin et du graphisme modifieraient la perception et la catégorisation selon la forme géométrique de tracés de différentes couleurs, tailles, et épaisseurs du trait. Dans le domaine musical, l'expertise est celle des musiciens qui ont de nombreuses années d'études et de pratique, qui ont appris à jouer d'un instrument et à décrire les structures musicales qu'ils perçoivent. Mais on peut se demander comme Bigand (2006) en quoi cette double compétence modifierait les processus d'écoute de la musique.

Dans cet article, nous rapportons deux expériences de catégorisation qui ont été 
menées pour tester la validité des UST à partir de leur pertinence cognitive. Il s'agit de tester des hypothèses qui découlent de la proposition d'UST comme figures sonométriques pour le temps, tout comme il existe des figures géométriques pour l'espace. En présentant à des auditeurs des segments musicaux variés qui relèvent d'UST et en leur demandant de les regrouper selon l'évolution temporelle du son, il se pourrait qu'il s'agisse d'une tâche difficile voire impossible, ou encore qu'il n'y ait aucune similitude entre les groupes réalisés par les participants. Un tel résultat ne serait pas compatible avec l'hypothèse de figures sonométriques. Il se pourrait que la tâche ne soit pas réalisable par des non musiciens, mais par des musiciens qui ont une pratique de la production de flux musicaux. L'hypothèse de figures sonométriques serait alors conditionnée par l'expertise. Si les groupes réalisés par les musiciens correspondent aux UST, l'hypothèse que les figures sonométriques soient des UST serait aussi conditionnée par l'expertise.

Il se pourrait toutefois que tout auditeur, musicien ou non musicien, arrive à regrouper les segments musicaux, avec une similitude entre les groupes réalisés par les participants. Un tel résultat serait compatible avec l'hypothèse de figures sonométriques. Enfin, si les groupes réalisés correspondent aux UST, on peut envisager que celles-ci relèvent de catégories communes, c'est-à-dire ne relevant pas de l'application d'un procédé ou d'un apprentissage, et qu'elles soient de possibles unités du traitement cognitif qui permettent d'appréhender le flux musical.

Les deux expériences ont été menées pour vérifier l'hypothèse que les participants regroupent les segments musicaux, que les groupements d'extraits musicaux correspondent aux UST et que la pratique musicale n'a pas d'effets sur le regroupement en UST.

La procédure expérimentale des deux expériences est basée sur une tâche de catégorisation libre qui est une des méthodes les plus utilisées pour étudier la catégorisation (voir Tijus et Cordier, 2003 pour une revue des méthodes d'investigation de la catégorisation). La méthode d'analyse est basée sur la matrice de confusion (Townsend, 1971 ; Luce \& Green, 1974 ; Nosofsky, 1992) et sur la construction de l'arbre de similarité Add tree (Sattath \& Tversky, 1977). La construction d'une matrice de confusion est une méthode classique de représentation des données dans les épreuves de détection, de reconnaissance, de catégorisation, et de classification ; par exemple en psychologie expérimentale pour étudier la reconnaissance de caractères (Tijus, 1986) ou de sons (McAdams, 1993) ou en intelligence artificielle pour l'apprentissage supervisé et l'évaluation des systèmes classificatoires. Dans la matrice, les colonnes correspondent aux réponses attendues et les lignes aux réponses observées. L'arbre de similarité correspond à un calcul de similarité entre éléments à catégoriser, à partir des groupes d'éléments dont chaque élément fait partie.

La première expérience est une investigation approfondie de la pertinence de quelques UST : le matériel sonore comprend 20 extraits sonores (4 segments musicaux pour chacune de 5 UST) qui sont catégorisés par 20 musiciens et 20 non musiciens. La deuxième expérience porte sur toutes les UST. Le matériel sonore 
comprend 76 extraits sonores (4 segments musicaux pour chacune des 19 UST) qui sont catégorisés par 19 musiciens et 19 non musiciens, chaque participant catégorisant 20 items (par tirage aléatoire de 4 segments musicaux pour chacune de 5 UST).

\section{EXPÉRIENCE 1}

L’objectif principal de l'expérience était d'observer avec quels autres segments musicaux un segment donné est regroupé pour vérifier que les segments relevant d'une même UST sont mis ensemble et qu'on n'observe pas de différence significative entre musiciens et non musiciens. S'agissant d'une première expérience, nous avons privilégié la redondance des observations (chaque segment est catégorisé 40 fois) plutôt que la variabilité (5 des 19 UST sont testées).

\section{MÉTHOde}

\section{- Participants}

L'expérience a été conduite auprès d'un groupe de 40 participants composé de 20 musiciens et de 20 non musiciens. Les non musiciens sont des étudiants et enseignants en psychologie n'ayant pas de pratique musicale. Les musiciens sont des adultes ayant au moins cinq ans de pratique musicale d'un instrument. Aucun participant ne connaissait les UST.

\section{- Matériel}

Le matériel expérimental est composé de 20 segments musicaux relevant des 5 UST, chaque UST étant représentée par 4 segments musicaux. Les 5 UST sont les suivantes : Trajectoire inexorable, Contracté-étendu, Qui avance, Obsessionnel et Qui tourne. Les segments musicaux sont des extraits d'œuvres musicales diverses ${ }^{1}$ sous la

(1) Pour chacune des 19 UST, les œuvres musicales desquelles sont extraits les segments expérimentauxutiliséspourlesexperiences1et2.Chute(Dutilleux, Ainsi la nuit : [1dutilleux], Gobin, Simulation: [1famine], Ton-That Tiét, Chu-Ky: [1ton-that], Machover, Famine: [1gobin]). Trajectoire inexorable(Debussy, La terrasse : [2levoile d'orphée], Frémiot, Ballade : [2stockhausen], Stockhausen, Telemusik: [2frémiot], Henry, Le voile d'Orphée: [2debussy])Élan(Dutilleux, Ainsi la nuit: [3scriabine], Mozart, Symphonie no 32 : [3dutilleux], Scriabine, Sonate $n^{\circ} 5:$ [3mozart], Teruggi, Xatys : [3terrugi]) Etirement(Anonyme, Flûte : [4debussy], Merlier, Ombres: [4variations], Debussy, Prélude à l'après midi d'un faune : [4anonyme], Henry, Variations pour une porte et un soupir : [4merlier]) Freinage(Levinas, Froissement d'ailes : [5levinas], Mion, Puzzlasept : [5reibel], Reibel, Variations en étoile: [5mion], Ferneyhough, Sonatas for string quartet: [5sonates]), Lourdeur(Merulo, Toccata seconda : [6murail], Murail, Territoires : [6merulo], Renouard La rivière : [Grenouard], Stravinsky, Le sacre du printemps: [6stravinsky]),Obsessionnel(Beethoven, Quatuor: [7beethovenr], Frémiot, Phonons. : [7frémiot], Riley, In C : [7yvanez], Yvanez, Sables: 
forme de fichiers de son. Les icônes de ces fichiers sont réparties aléatoirement dans une fenêtre de l'ordinateur. Les icônes, qui ont comme nom un numéro de 1 à 20 tiré au hasard, sont déplaçables dans le cadre de la fenêtre. Il est ainsi possible de les regrouper ou de les séparer. Pour écouter un segment musical, il faut cliquer sur l'icône.

\section{- Procédure}

La passation est individuelle. Le participant est assis face à l'ordinateur (Macintosh G5) et la consigne ${ }^{2}$ lui demande d'écouter successivement chacun des 20 segments musicaux

[7riley]),En suspension(Beethoven, Sonate à Kreutzer : [8risset], Rachmaninov, Concerto pour piano $n^{\circ} 3$ : [8beethoven], Risset, Songes : [8rachmaninov], Berio, Différences : [8différences]) Par vagues(Bach, Prélude en ré : [9sequenza], Bayle, Les couleurs de la nuit: [9bach], Berio, Sequenza III : [9bayle], Bryars, The Old Tower of Löbenicht: [9theoldtower]),Stationnaire(Bach, Prélude en ré : [10redolfi], Redolfi, Sunny afternoon at Bird Rock beach : [10etude], Renouard, Tchernoziom : [10bach], Ligeti, Étude no 2 pour piano : [10renouardl]), Contracté-Étendu (Beethoven, trio des esprits : [11beethoven], Gobin, What objects are the fountains of the happy strain ?: [11prod'homme], Parmegiani, De natura sonorum : [11parmegiani], Prod'homme, Jeux: [11gobin]),Qui avance(Chabrier, Scherzo valse.wav: [12parmegiani], Facoli, Aria: [12malboscsuite], Malbosc, Suite guitare.wav : [12facoli], Parmegiani, La roue Ferris : [12chabrier]), Qui veut démarrer(Debussy, Laterrasse:[13merlier], Frémiot, Tsim' ballade:[13debussy], Merlier, La grotte:[13mozart],MozartSymphonien41:[13frémiot])Suspension-Interrogation(Beethoven, Sonateà Kreutzer:[14malboscsuite], Debussy, Laterrasse:[14beethoven], Malbosc, Faisceau parallèle: [14debussy], Malbosc, Suite : [14malboscfaisceau]), En flottement (Bayle, Théatre d'ombres: [15parmegiani], MIM, En flottement: [15redolfi], Parmegiani, Violostries : [15mim], Redolfi, Pacific Tubular Waves: [15bayle]),Sans direction divergenc(Fourès, Trafic: [16stockhausen], Parmegiani, L'enfer: [16pascal], Pascal, Puzzle : [16parmegiani], Stockhausen, Telemusik: [16fourès]), Qui tourne(MIM, Qui tourne: [17mim], Die Tanzlustigen : [17schaeffer], Schaeffer, Étude violette : [17dietanzlustigen], Takemitsu, Water Music. : [17watermusic]),Sans direction excès (Anonyme, impro: [18maleclumina], Frémiot, flux : [18anonymeimpro], Lejeune, Invitation : [18fremiotflux], Maleclumina : [18lejeuneinvitation])Sur l'erre(Fouad, Thaksim : [19schumann], Merlier, La harpe : [19merlier], Murail, Territoires : [19murail], Schumann, Arabeske : [19fouad]). (2) Consignedesexpériences1et2.

Ilexistedenombreusesfaçonsd'écouteretderessentirlamusique.Ainsi,onpeutsefocalisersur l'émotionqu' ellevéhicule sursonrythme,sontimbresul'harmonie,ouencoresur'instrumentation, oul'époqueàlaquelleelleaétécomposée.Danscetteexpérience, ilvousestdemandédevous focalisenuniquementsurledéroulementtemporeldelamatièresonore.

- Dansunpremiertemps,vousallezécouterattentivement'ensembledes20extraitsmusicauxafin devousfaireuneidéedesdifférencesquiexistententreeux.

-Vousallezensuitelesclasserenfonctiondelafaçondontlamatièresonorévoluedandetemps. Vousrassemblerezles extraitsquisuiventlemêmedéroulementtemporelengroupes, chaque groupecorrespondantàuneclassededéroulementtemporel.Sivousn'êtespassûr(e)devotre classement,vouspourrezréécouterchaqueextrait,etchangersonclassementautantdefoisqu'il vousconvient.

Si vous le souhaitez, vous pouvez faire une petite pause à n'importe quel moment. 
et de les rassembler ou de les séparer pour constituer des groupes (catégorisation libre) en faisant attention au déroulement temporel des segments musicaux.

Lorsqu'un participant estime avoir accompli la tâche de groupement, une copie d'écran est faite et il lui est demandé d'entourer les groupes constitués, de les nommer si possible, et de justifier les groupements réalisés. La tâche dure environ 45 minutes.

\section{- Unités d'analyse}

Les catégories réalisées par les participants ont été analysées et confrontées à la catégorisation théorique des UST. Pour cela, les données ont été traitées à partir de la matrice de confusion de $20 \times 20$ segments musicaux et en calculant la fréquence d'occurrence avec laquelle deux segments sont regroupés: pour chaque paire de segments musicaux, nous notons [1] lorsque les extraits sont mis ensemble et [0] dans le cas contraire. Les matrices de confusion des musiciens et des non musiciens ont ensuite été calculées en faisant la somme des matrices individuelles. Ces fréquences ont ensuite été moyennées dans une matrice de confusion $5 \times 5$ correspondant aux fréquences avec lesquelles les 4 segments d'une UST sont distribués dans les 5 UST.

La matrice de confusion obtenue est confrontée à la matrice de confusion théorique de la répartition des segments musicaux en UST et à la matrice de répartition aléatoire. Pour celle-ci, chaque participant ayant à réaliser des groupes à partir de 4 segments pour chacune des 5 UST, la probabilité de grouper aléatoirement un segment d'une UST avec un des quatre segments d'une autre UST est de .21 (4/19) et de .16 (3/19) pour la probabilité de grouper un segment avec un des trois autres segments de la même UST. Enfin, le regroupement des segments musicaux a aussi été analysé à partir de la construction d'arbres de similarité selon la méthode Add tree (Sattath \& Tversky, 1977) pour être confronté aux UST.

\section{Résultats}

La Figure 1 donne un exemple de réalisation finale pour un participant. Les segments musicaux relèvent de 5 UST, avec 4 segments par UST. Les participants font en moyenne 6,57 groupes, soit un peu plus de groupes que d'UST. En moyenne, les non musiciens font 6,4 groupes et les musiciens 6,75 groupes. Cette différence n'est pas significative, $F(1,38)=.19$; ns.

\footnotetext{
- Àlafindevotreclassement,réécouteztouslesextraitsdechaquegroupepourvérifiervotre groupement. Ajustez les classements une dernière fois, si nécessaire. Il vous sera demandé d'expliquerlaformedudéroulementtemporelquicaractérisechaqueclasse,etdelanommer. -VousbénéficiezdutempsquevousvoulezN'hésitexpas̀poserdesquestionsd'expérimentateur siquelquechosen'estpasclaire.
}

Nous vous remercions pour votre participation à cette expérience. 


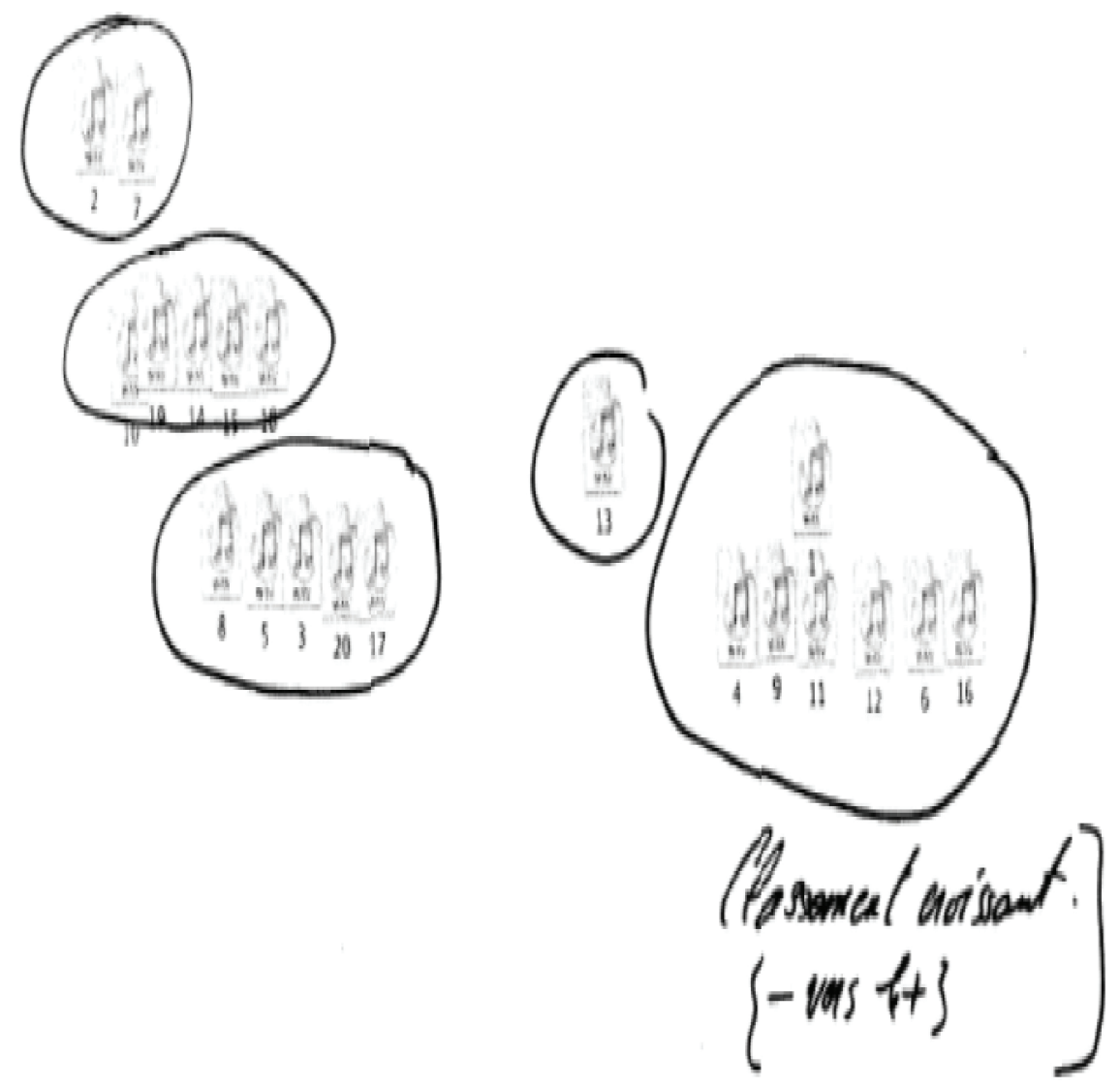

Figure 1.

Exemple de réalisation de la répartition de 20 extraits musicaux par un participant.

Les données du Tableau 1 montrent que les participants ont tendance à regrouper ensemble les segments musicaux qui appartiennent à une même UST : la fréquence moyenne de segments musicaux mis ensemble appartenant à la même UST (.38) est supérieure à la fréquence moyenne de segments musicaux mis ensemble appartenant à des UST différentes $(.16), F(1,38)=306 ; p<.0001$.

Le Tableau 2 donne les résultats pour les musiciens et les non musiciens. La différence entre musiciens (.36) et non musiciens (.39) n'est pas significative, $F(1,38)$ $=.51 ; \mathrm{ns}$. Il n'y a pas non plus d'interaction entre musiciens et non musiciens et la distribution des segments musicaux en UST, $F(24,38)=1.17$; ns.

Les participants groupent un segment musical plutôt avec les 3 autres segments de la même UST, bien que la probabilité soit plus grande de grouper avec les 4 segments d'une autre UST. La probabilité de grouper au hasard un segment avec l'un des 3 segments de la même UST est de .16 (3/19) contre .21 (4/19) avec l'un 
Tableau 1

Pourcentages d'association pour chacun des 20 segments musicaux avec les 19 autres segments selon I'UST cible d'appartenance. Chaque cellule correspond à 160 observations : chacun des 40 participants a groupé (1) ou n'a pas groupé (0) le segment musical (en ligne) avec l'un des 4 segments de I'UST (en colonne).

\begin{tabular}{lllllll} 
& & \multicolumn{5}{c}{ UST cible } \\
\cline { 3 - 7 } & & contract-ét & obsessionnel & qui avance & qui tourne & traj inex \\
\hline beethov-trio & contract-ét & 0,44 & 0,17 & 0,15 & 0,09 & 0,15 \\
gobin-object & contract-ét & 0,40 & 0,11 & 0,14 & 0,15 & 0,21 \\
parme-denatu & contract-ét & 0,28 & 0,05 & 0,15 & 0,20 & 0,32 \\
prod-hom jeu1 & contract-ét & 0,50 & 0,09 & 0,09 & 0,13 & 0,19 \\
becth-quatuo & obsessionncl & 0,10 & 0,34 & 0,26 & 0,19 & 0,11 \\
frém-phonon & obsessionnel & 0,05 & 0,34 & 0,20 & 0,26 & 0,14 \\
riley-in & obsessionnel & 0,12 & 0,37 & 0,09 & 0,28 & 0,14 \\
yvanez-sable & obsessionnel & 0,08 & 0,37 & 0,13 & 0,20 & 0,21 \\
chabrier-vals & qui avance & 0,14 & 0,20 & 0,44 & 0,11 & 0,10 \\
facoli-aria & qui avance & 0,14 & 0,17 & 0,47 & 0,11 & 0,11 \\
malb-suit guit & qui avance & 0,37 & 0,11 & 0,31 & 0,10 & 0,10 \\
parm-la roue & qui avance & 0,09 & 0,20 & 0,35 & 0,23 & 0,13 \\
bayle-t-ombr & qui tourne & 0,08 & 0,24 & 0,12 & 0,41 & 0,16 \\
malb-paradis & qui tourne & 0,11 & 0,21 & 0,14 & 0,37 & 0,18 \\
mim-q-tourn & qui tourne & 0,06 & 0,25 & 0,15 & 0,40 & 0,14 \\
scaff-E-violl & qui tourne & 0,13 & 0,22 & 0,13 & 0,37 & 0,15 \\
debus-terras & traj inex & 0,25 & 0,12 & 0,14 & 0,12 & 0,37 \\
frém-balad & traj inex & 0,16 & 0,22 & 0,17 & 0,13 & 0,32 \\
prod-hom jeu & traj inex & 0,18 & 0,19 & 0,09 & 0,25 & 0,28 \\
stock-telem & traj inex & 0,14 & 0,14 & 0,10 & 0,22 & 0,39 \\
\hline
\end{tabular}

des 4 segments d'une autre UST, dans la mesure où la probabilité de classer un extrait avec un extrait de la même UST (trois autres extraits) n'est pas la même que celle de le classer avec une autre UST (quatre autres extraits). Si la probabilité avait été la même, on peut estimer par proportionnalité que la fréquence observée du regroupement d'un segment avec un autre segment aurait été de .44 lorsqu'il s'agit de la même UST contre .14 lorsqu'il s'agit d'une autre UST.

Les segments musicaux ne sont pas pareillement attribués à leur UST. Certaines UST sont mieux appréhendées que d'autres: les segments musicaux de l'UST Qui avance sont plus souvent mis ensemble que ceux de l'UST Trajectoire inexorable par exemple. Enfin, certains segments musicaux sont plus typiques de leur UST d'appartenance que d'autres. Ainsi, pour l'UST Qui avance, l'extrait de Marco Venetiano Facoli : Aria della Marchetta Schiavonetta est plus associé aux autres segments de la même UST que l'extrait de Pierre Malbosc : Suite pour guitare.

L'analyse hiérarchique, donnée dans la Figure 2, à partir des groupements réalisés par les participants, montre que les UST Contracté-étendu, Qui avance, 
Tableau 2

Pourcentages d'association pour les 4 segments musicaux d'une UST avec les 4 segments musicaux de chacune des cinq UST, pour les musiciens et les non musiciens. Chaque cellule correspond à 80 observations : chacun des 20 participants, musiciens ou non musiciens, a groupé (1) ou n'a pas groupé (0) chacun des 4 segments musicaux d'une UST (en ligne) avec les 4 segments de I'UST (en colonne).

\begin{tabular}{lccccc} 
& \multicolumn{5}{c}{ UST cible } \\
\cline { 2 - 6 } & \multicolumn{5}{c}{ non-musiciens } \\
\cline { 2 - 6 } UST du Segment & contract-ét & qui avance & qui tourne & obsessionnel & traj inex \\
\hline contract-ét & 0,40 & 0,10 & 0,13 & 0,12 & 0,24 \\
qui avance & 0,12 & 0,41 & 0,16 & 0,17 & 0,14 \\
qui tourne & 0,13 & 0,13 & 0,38 & 0,22 & 0,15 \\
obsessionnel & 0,12 & 0,15 & 0,23 & 0,35 & 0,15 \\
traj inex & 0,25 & 0,13 & 0,16 & 0,15 & 0,31 \\
\hline & & & musiciens & & \\
UST du Segment & contract-ét qui avance & qui tourne & obsessionnel & traj inex \\
\hline contract-ét & 0,55 & 0,13 & 0,10 & 0,10 & 0,13 \\
qui avance & 0,12 & 0,42 & 0,15 & 0,20 & 0,11 \\
qui tourne & 0,08 & 0,14 & 0,37 & 0,24 & 0,17 \\
obsessionnel & 0,08 & 0,19 & 0,24 & 0,33 & 0,15 \\
traj inex & 0,15 & 0,12 & 0,23 & 0,18 & 0,32 \\
\hline
\end{tabular}

inexorable et Qui tourne sont relativement bien discriminées, ce qui n'est pas le cas de l'UST Obsessionnel.

Enfin, le Tableau 1 montre qu'à partir des données comportementales de groupements réalisés par les participants, partant d'un segment musical, on retrouve l'UST d'appartenance, sauf pour deux des vingt segments. Le segment musical extrait de Bernard Parmegiani : De natura sonorum de l'UST Contracté-étendu semble plutôt relever pour les participants de l'UST Trajectoire inexorable et le segment Pierre Malbosc : Suite pour guitare de l'UST Qui avance relèverait plutôt pour les participants de l'UST Contracté-étendu. Pour les 18 autres segments (90\%), les données comportementales de groupe permettent d'inférer l'UST d'appartenance.

\section{Discussion}

Cette première expérience sur la validité psychologique des UST, comme figures temporelles sonores, ne portait que sur 5 UST. Un premier résultat est que les participants, musiciens ou non, semblent bien percevoir des formes temporelles qui 


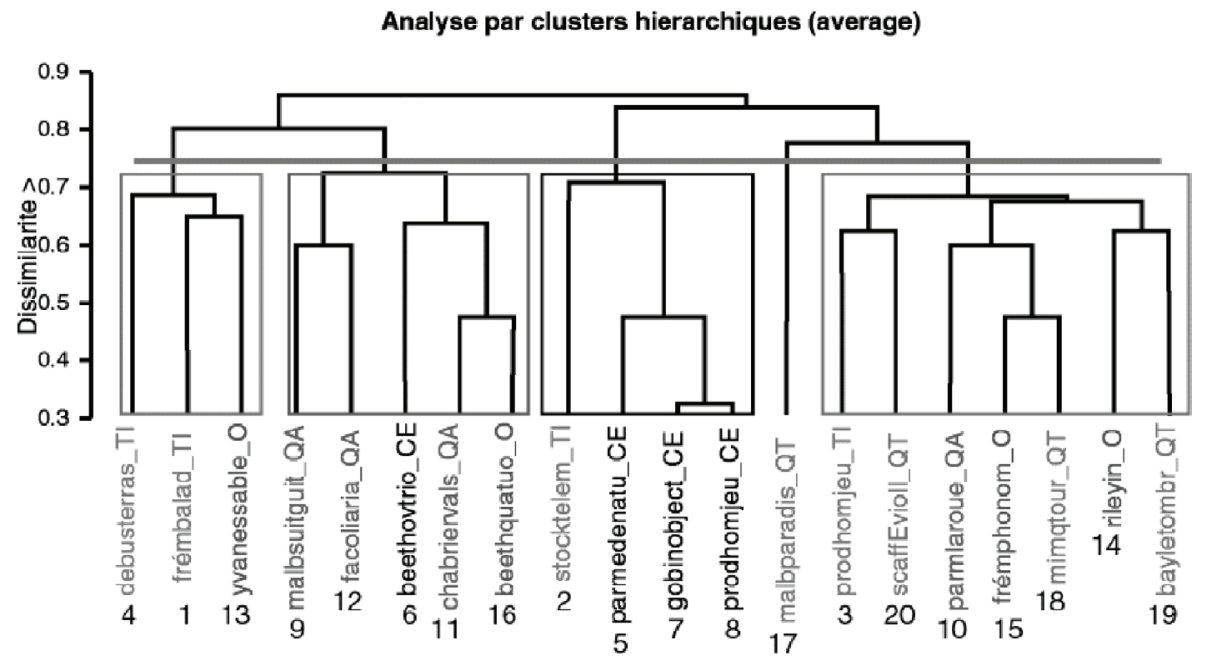

Figure 2.

Arbre hiérarchique de similarité résultant de l'analyse des associations observées auprès des musiciens et des non musiciens pour l'expérience 1 portant sur 5 UST : Trajectoire inexorable (segments 1 à 4), Contracté-Étendu (segments 5 à 8), Qui avance (segments 9 à 12), Obsessionnel (segments 13 à 16) et Qui tourne (segments 17 à 20).

leur permettent de regrouper les segments musicaux et aucun d'entre eux n'a jugé la tâche trop difficile pour être réalisée.

Un second résultat est que les segments musicaux correspondant à une même UST sont majoritairement regroupés ensemble. En d'autres termes, parmi les associations réalisées, le regroupement des segments musicaux en UST est le type d'association le plus élevé.

Enfin, les musiciens et les non musiciens font en moyenne le même nombre de groupes et, dans la constitution des groupes selon les UST, la différence entre musiciens et non musiciens n'est pas significative. La raison pourrait bien être que l'expertise nécessaire soit une expertise liée à l'écoute musicale, celle-ci étant largement partagée, qu’on soit musicien ou non.

\section{EXPÉRIENCE 2}

Les résultats de l'expérience 1 ont motivé la réalisation d'une deuxième expérience, en considérant l'ensemble des 19 UST et en programmant un logiciel dédié à la réalisation de groupements de segments musicaux. Avec l'expérience 1, écouter un segment musical consistait à cliquer sur son icône, ce qui avait comme effet d'ouvrir le fichier son qu'il fallait ensuite activer. Le logiciel dédié de l'expérience 2 facilite l'écoute des segments musicaux. 


\section{- Participants}

L'expérience a été conduite auprès d'un groupe de 38 participants (19 musiciens et 19 non musiciens). Les non musiciens sont des étudiants et enseignants en psychologie n'ayant pas de pratique musicale. Les musiciens sont des adultes ayant au moins cinq ans de pratique musicale d'un instrument. Aucun participant ne connaissait les UST.

\section{- Matériel}

Le matériel provient de la base de données d'exemples de segments musicaux, extraits d'œuvres musicales variées, catégorisés en UST par le MIM. Les segments musicaux utilisés ont été tirés au hasard parmi ces exemples à raison de 4 extraits par UST, soit un total de 76 extraits.

L'expérience se déroule individuellement dans une cabine audiométrique. Les participants écoutent les extraits avec un casque. L'expérience est guidée par ordinateur (Macintosh) avec le logiciel de passation "Catégolibre» développé dans le langage de programmation MATLAB. Les données des participants sont recueillies automatiquement avec la constitution d'un fichier sous forme de listes directement exploitables pour être transformées en matrices.

Les segments musicaux sont présentés sous la forme de fichiers de son, avec leur icône disposée aléatoirement dans un espace à deux dimensions. Les icônes, qui ont comme nom un numéro tiré au hasard entre 1 et 20 , sont déplaçables dans la fenêtre de l'ordinateur : il est ainsi possible de les regrouper ou de les séparer. Pour écouter un segment, il faut cliquer sur l'icône.

\section{- Procédure}

Pour éviter à chaque participant l'écoute et la catégorisation de 76 segments musicaux, - ce qui aurait demandé trop d'attention et trop de temps, les extraits pouvant durer plus de 30 secondes —, chaque participant a eu 20 segments à catégoriser, 4 segments par UST pour 5 UST. Pour cela, la distribution des 76 extraits entre chacun des 19 musiciens et des 19 non musiciens a été réalisée de manière aléatoire et sans remise en 19 listes de 5 UST, chaque UST étant représentée par 4 segments pour 5 UST.

Avec la consigne ${ }^{3}$, il est demandé à chaque participant d'écouter chacun des 20 segments musicaux, autant de fois qu'il le souhaite, et de constituer des groupes (catégorisation libre) selon le déroulement temporel des segments musicaux. La tâche dure environ 45 minutes.

(3) Voir consigne note 2, p. 420. 


\section{- Unités d'analyse}

Les unités d'analyse sont celles de l'expérience 1 pour une première matrice de confusion de $76 \times 76$ segments musicaux et une deuxième matrice de confusion $19 \times 19$ UST correspondant aux fréquences avec lesquelles les 4 segments d'une UST sont distribués dans les 19 UST. Un coefficient de corrélation entre les matrices de confusion des musiciens et des non musiciens a été calculé en appliquant le test de Mantel (1967). Ce test est basé sur des permutations aléatoires, et permet d'observer la significativité de la corrélation entre les matrices de confusion obtenues différemment sur les mêmes objets. Enfin, comme pour l'expérience 1, le regroupement des segments en UST a aussi été analysé avec les arbres de similarité selon la méthode Add tree (Sattath \& Tversky, 1977).

\section{Résultats}

Dans l'expérience 2, conformément aux résultats de l'expérience 1 qui ne portaient que sur 5 UST, on s'attend pour les 19 UST (i) à ce que les participants fassent des regroupements qui correspondent aux UST, et (ii) à ne pas observer de différences entre musiciens et non musiciens, la catégorisation de segments musicaux en figures temporelles sonores ne relevant pas de l'expertise.

Un premier résultat concerne le nombre de groupes réalisés par les participants. Chaque participant ayant 4 segments musicaux relevant de 5 des 19 UST, le nombre de groupes cibles est de 5. En moyenne, les non musiciens font 5,7 groupes et les musiciens 5,9 groupes. Comme pour l'expérience 1, cette différence n'est pas significative $(p=.62)$. Le nombre de groupes est ici plus proche du nombre d'UST, ce qui peut être un effet de la facilitation apportée à la tâche de catégorisation par l'utilisation d'un logiciel dédié à l'écoute et à la catégorisation de segments musicaux. Pour le regroupement des segments d'une même UST, il n'y a pas non plus de différences entre la réussite des musiciens (.36) et celle des non musiciens (.38), $F_{1,36}=.51 ; \mathrm{ns}$. Enfin, la corrélation entre les données des musiciens et celles des non musiciens étant significative $(r=.43, p<.001)$, pour les analyses suivantes, leurs données ont été regroupées.

Comme pour l'expérience 1, dans la mesure où la probabilité de grouper un extrait avec un extrait de la même UST (trois autres extraits) n'est pas la même que celle de le grouper avec une autre UST (quatre autres extraits), les données ont été ajustées avant d'être analysées. Pour cela, les résultats de la matrice de confusion ont été convertis en fréquence relative, et sachant qu'un extrait a seulement 3 chances d'être classé avec un extrait provenant de la même UST (puisqu'il ne peut pas être classé avec lui-même) contre 4 chances d'être classé avec un extrait d'une autre UST, les valeurs de la matrice des probabilités de tirage aléatoire sont soustraites aux valeurs correspondantes de la matrice de confusion. Les analyses sont effectuées sur la matrice résultante, à partir des fréquences relatives. 
Le résultat est que les segments musicaux d'une même UST se retrouvent plus souvent groupés ensemble qu'avec des segments d'une autre UST. Pour chacune des 19 UST, les participants ont plutôt regroupé leurs segments musicaux : il y a une différence significative entre l'association avec des segments de la même UST et l'association avec des segments d'une autre UST (Tableau 3).

Tableau 3

Pour chacune des 19 UST, table des F des différences de groupement des segments de cette UST avec des segments de toutes les UST. Les segments d'une même UST sont significativement mis ensemble.

\begin{tabular}{lcc}
\hline UST & F Ratio & Prob $>$ F \\
\hline Chute & 20,84 & $<, 0001$ \\
Trajectoire inexorable & 12,06 & $<, 0001$ \\
Elan & 13,71 & $<, 0001$ \\
Etirement & 17,98 & $<, 0001$ \\
Freinage & 27,76 & $<, 0001$ \\
Lourdeur & 5,77 & $<, 0001$ \\
Obsessionel & 8,64 & $<, 0001$ \\
En suspension & 13,34 & $<, 0001$ \\
Par vagues & 6,00 & $<, 0001$ \\
Stationnaire & 10,90 & $<, 0001$ \\
Contracté-étendu & 47,26 & $<, 0001$ \\
Qui avance & 6,83 & $<, 0001$ \\
Qui veut démarrer & 10,60 & $<, 0001$ \\
Suspension-interrogation & 8,16 & $<, 0001$ \\
En flottement & 13,11 & $<, 0001$ \\
Sans direction par divergence d'informations & 8,90 & $<, 0001$ \\
Qui tourne & 26,66 & $<, 0001$ \\
Sans direction par excès d'informations & 39,56 & $<, 0001$ \\
Sur l'erre & 13,59 & $<, 0001$ \\
\hline
\end{tabular}

La Figure 3 présente pour chaque groupe de 4 segments musicaux constituant une UST (en ligne), la moyenne des fréquences de regroupement avec les autres segments musicaux des 19 UST (en colonne). Une valeur proche de zéro signifie un choix au hasard, une valeur positive une tendance à choisir l'UST, une valeur négative une tendance à ne pas la choisir l'UST. On observe pour chaque UST (en ligne) la distinction d'une ou plusieurs UST (en colonne). Ainsi, pour l'UST Chute, les segments de cette UST ont été classés majoritairement ensemble. En revanche, si 


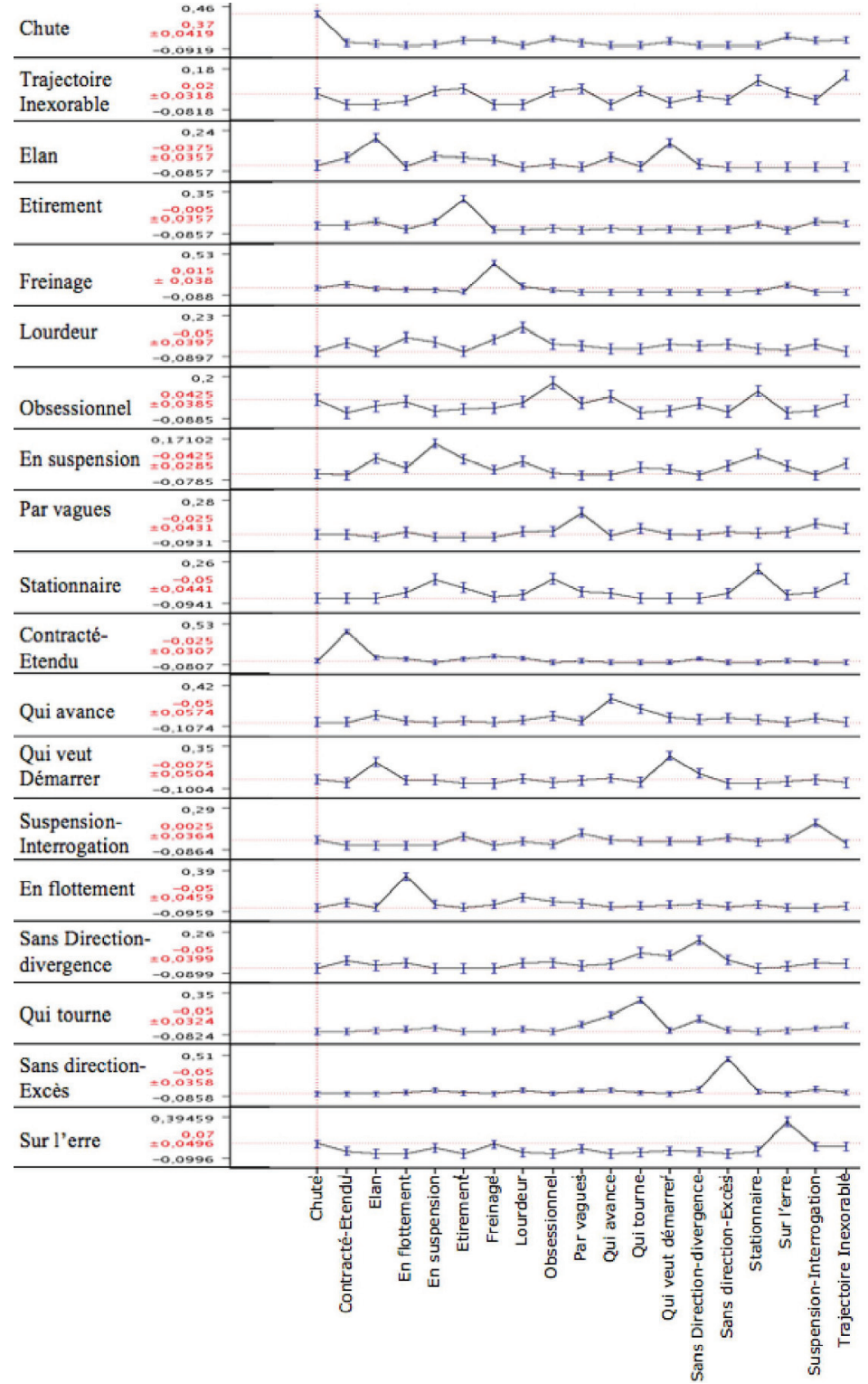

Figure 3.

Pour chaque groupe de 4 segments musicaux d'une UST (en ligne), la moyenne des fréquences de regroupement avec les autres segments musicaux selon leur UST (en colonne). Cette moyenne est diminuée de la probabilité de choisir au hasard un segment d'une UST, soit (3/76) quand il s'agit de la même UST en ligne et en colonne, soit (4/76) autrement. Une valeur proche de zéro signifie un choix au hasard, une valeur positive une tendance à choisir l'UST correspondante, une valeur négative une tendance à ne pas choisir I'UST correspondante. Les pointillés indiquent la valeur obtenue pour l'UST Chute. 
les segments qui relèvent de l'UST Obsessionnel ont tendance à être regroupés ensemble, ils montrent aussi une tendance à être mis avec des segments des UST Stationnaire et de Qui Avance.

Avec les groupements, les segments de certaines UST sont plus associés entre eux que ceux d'autres UST. Certaines UST sont ainsi mieux réalisées que d'autres : par exemple, les segments de l'UST Chute sont associés entre eux dans une proportion de .46 contre .18 pour l'UST Trajectoire Inexorable. La première a un taux de regroupement supérieur à la deuxième. Toutefois, en considérant les segments musicaux comme variable aléatoire, l'ANOVA montre que les différences entre UST ne sont pas significatives $\left(F_{18,57}=1,62 ; p=.08\right)$ : certaines UST ne sont pas significativement mieux reconnues que d'autres.

Pour chacune des 19 UST, en comparant le taux de regroupement des segments dans leur UST cible au taux de dispersion dans d'autres UST, l'ANOVA montre que les segments les mieux catégorisés en USTs sont les segments des UST Chute $(t=18.5 ; p<.0001)$, Freinage $(t=20.43 ; p<.0001)$, Etirement $(t=16.26$; $p<.0001)$, En Flottement $(t=14.30 ; p<.0001)$, Sans Direction Par Excès $(t=26.01$; $p<.0001)$, Contracté Étendu ( $t=27.87 ; p<.0001)$, Sur l'erre $(t=14.03 ; p<.0001)$. Pour ces UST, la différence entre regroupement en UST cible et dispersion dans d'autres UST est importante $(t>10)$. Les segments des UST Lourdeur $(t=7.46$; $p<.0001)$, Par Vagues $(t=8.25 ; p<.0001)$, Suspension-Interrogation $(t=9.39$; $p<.0001)$ sont moins distingués, mais de manière significative. Pour les autres UST que sont Stationnaire, Obsessionnel, En Suspension, Trajectoire Inexorable, Qui tourne, Qui avance et Sans direction par divergence, leurs segments ne sont pas regroupés de manière significative dans l'UST cible, mais celle-ci est toujours celle qui recueille le plus fort taux de regroupement.

Le regroupement des segments musicaux en UST a aussi été analysé à partir de la construction d'un arbre de similarité. Cette analyse par clusters (Figure 4) montre deux grands groupes, le premier est composé de 2 sous-groupes et le deuxième de trois sous-groupes. Au niveau 3, on obtient 19 groupes, ce qui correspond au nombre d'UST établit par le MIM. La Figure 4 montre que les extraits appartenant aux UST Sans direction par excès d'information, En flottement, Étirement, Sur l'erre, Freinage et Contracté Étendu sont regroupés. Ce résultat corrobore les résultats de l'ANOVA : les UST les mieux reconnues sont celles que l'on retrouve groupées dans l'arbre de similarité.

\section{Discussion}

Un premier résultat de cette deuxième expérience est que, comme pour l'expérience 1, les participants (qu'ils soient musiciens ou non) perçoivent bien des formes temporelles qui leur permettent de regrouper les segments musicaux. Il est ici aussi notable que musiciens et non musiciens font en moyenne le même nombre de 


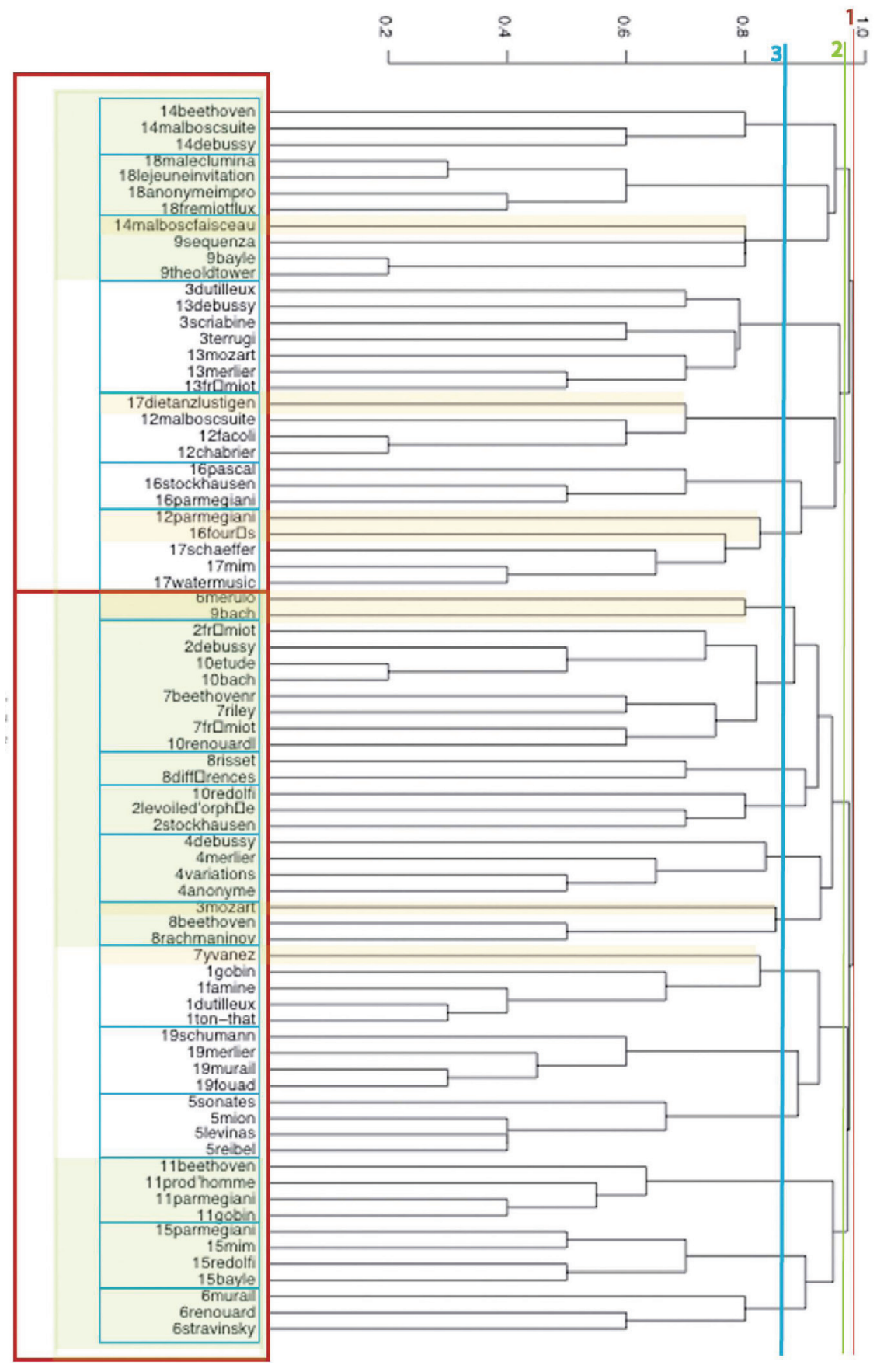

Figure 4.

Arbre hiérarchique de similarité résultant de l'analyse des associations observées auprès des musiciens et des non musiciens pour l'expérience 2 portant sur les 19 UST. 
groupes et que dans la constitution des groupes selon les UST, la différence entre musiciens et non musiciens ne soit pas significative. La raison pourrait bien être que l'expertise nécessaire soit une expertise liée à l'écoute, celle-ci étant largement partagée, qu'on soit musicien ou non.

Le second résultat est que les segments correspondant à une même UST sont, comme dans l'expérience 1, significativement regroupés ensemble. Ainsi, parmi les associations réalisées en regroupant des segments musicaux, le regroupement selon l'UST cible avec une faible dispersion dans d'autres UST est le type d'association le plus fréquent. Enfin, même si certains segments musicaux ne sont pas groupés avec les autres segments de la même UST, avec une variabilité entre UST, les groupements selon les UST semblent former des groupes cohérents.

Les participants se sont ainsi montrés capables de percevoir le déroulement d'extraits sonores sous la forme de figures temporelles sonores et ont classé ces extraits selon des catégories de mouvement. Alors que la consigne ne stipulait pas de dessiner, la Figure 5 montre un des dessins (participant no ${ }^{\circ}$ ) réalisés spontanément par certains des participants. Ces dessins ont peut-être aidé à comparer le déroulement temporel des segments musicaux. En représentant le déroulement temporel de la matière sonore par des courbes ou des figures sur le papier, ces participants ont effectué une schématisation spatiale des données temporelles en résolvant la contrainte du temps par un codage analogique spatial. Par exemple, le participant 17, qui a numéroté les 19 dessins correspondants aux 19 segments musicaux qu'il devait catégoriser, a produit des dessins 1, 5, 12, 18 qui sont similaires et qui pour trois d'entre eux correspondent à l'UST Qui tourne. Les dessins 4, 7 et 16 qui se ressemblent correspondent à l'UST Qui avance.

\section{DISCUSSION GÉNÉRALE ET CONCLUSION}

Les deux expériences ont été menées pour vérifier l'hypothèse que les participants regroupent les segments musicaux, que les groupements d'extraits musicaux correspondent aux UST et que la pratique musicale n'a pas d'effets sur le regroupement en UST. Les résultats montrent que les auditeurs, musiciens et non musiciens, arrivent à regrouper les segments musicaux selon leur déroulement temporel, avec une similitude entre les groupes réalisés par les participants. Un tel résultat est compatible avec l'hypothèse de figures sonométriques. Enfin, les groupes réalisés sont compatibles avec l'hypothèse des UST, comme figures sonométriques communes, c'est-à-dire ne relevant pas de l'application d'un procédé ou d'un apprentissage.

Les résultats confortent l'idée que les auditeurs perçoivent les UST à travers les segments musicaux, tout comme on peut percevoir un triangle dans la photographie du toit en pente d'une maison. De plus, le fait que les musiciens ne réussissent pas mieux cette tâche que les non musiciens renforce l'hypothèse que cette compétence relève, soit de prédispositions humaines, soit d'un apprentissage naturel basé sur l'extraction des régularités dans l'évolution temporelle du flux sonore. 


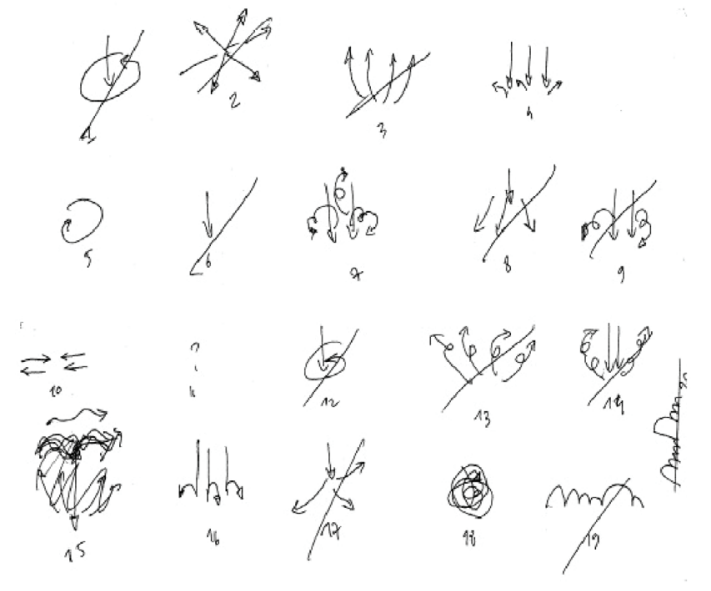

\begin{tabular}{|c|c|}
\hline 1:12pamegiani & Qhicuxance \\
\hline 2:I lopascal & Sans direction divergence \\
\hline 3:18malechumina & Sans direction excès \\
\hline 4:I2facoli & Quianance \\
\hline 5:17dietanzhstigen & Quitoume \\
\hline 6:17watermusic & Quitoume \\
\hline 7:I2chabrier & Qhianance \\
\hline 8:I6fourès & Sans direction divergence \\
\hline 9:18anornsmeimpro & Sans directionexcès \\
\hline 10: Ioparmegiani & Sans direction divergence \\
\hline 11:16stockhausen & Sans direction divergence \\
\hline 12:17schaeffer & Quitoume \\
\hline 13: 18legenmeimntation & Sans directionexcès \\
\hline 14: 14beethoven & Suspension-Intenvgation \\
\hline 15: 14malbascfaiscean & Suspension-Intenvgation \\
\hline 16:12malboscsuite & Quianance \\
\hline 17: I8fiemiotflux & Sans directionexcès \\
\hline 18:17mim & Quitoume \\
\hline 19: 14debuasyy & Suspension-Intenvgation \\
\hline 20: I4malbascsuate & Suspension-Inteno. \\
\hline
\end{tabular}

Figure 5.

Détail d'une feuille de dessin utilisée par le participant 17, non musicien, lors de la passation de l'expérience pour coder les segments musicaux.

Les performances des auditeurs sont notables. Ainsi, en considérant un groupe de segments musicaux et en demandant à des auditeurs de les grouper selon leur déroulement temporel, comme lors des expériences 1 et 2, les groupes réalisés vont correspondre aux UST. Nous avons pu estimer à .44 la fréquence de regroupement des segments musicaux en UST. Il s'ensuit que le tirage au hasard d'un des segments d'un regroupement réalisé par un participant donnerait une probabilité moyenne de .44 d'avoir choisi un segment qui correspond effectivement à l'UST.

La catégorisation en UST n'est toutefois pas optimale. Plusieurs raisons peuvent être avancées. Une première raison tient aux conditions d'observation: avec l'expérience 2, en facilitant avec un logiciel dédié les conditions de passation, moins d'actions à faire et un délai plus court pour écouter un segment musical, par rapport à l'expérience 1, le regroupement en UST s'est trouvé renforcé alors même que les observations portaient sur la totalité des UST. Une deuxième raison tient au manque d'expérience de l'explicitation de figures temporelles sonores et au manque de mots pour les dénommer. Si les auditeurs ont un savoir sur les UST, ce savoir est un savoir implicite. Une troisième raison peut être la difficulté intrinsèque à certaines catégories pour des raisons de complexité cognitive. Par analogie avec les figures géométriques planes, on conçoit bien que les trapèzes sont des figures plus complexes que les rectangles et qu'il soit plus difficile de différencier un carré d'un losange, qu'un carré d'un cercle. Cette source de difficulté est relative à l'ensemble des catégories que sont les USTs et à la structure de cet ensemble de catégories. Les USTs sont considérées 
comme indépendantes, alors qu'elles partagent des descripteurs en commun. Il devrait y avoir une hiérarchie des catégories des figures temporelles sonométriques tout comme il y a une hiérarchie des figures géométriques planes dans laquelle les carrés sont des rectangles particuliers. On trouve de tels exemples dans les données des participants. Ainsi les segments trajectoire inexorable sont souvent mis avec des segments qui avance, mais pas l'inverse. Le travail de mise en relation des USTs est un travail en cours qui est facilité par les résultats expérimentaux. Enfin, une dernière raison peut relever de la définition des UST. Si la formalisation des UST par le MIM est bien avancée, elle n'est pas terminée. Les résultats des expérimentations servent ici aussi à mieux définir les UST en s'interrogeant sur les segments les moins bien catégorisés en UST.

Les résultats sont toutefois encourageants car il ne faut pas considérer la recherche sur les USTs comme un travail achevé, mais comme relevant du produit d'une démarche scientifique. Les chercheurs du MIM ont cherché à définir un petit nombre de figures temporelles qui permettent de découper tout flux sonore. Ce travail de typologie est fastidieux. Lorsqu'un élément musical ne correspond pas à la catégorisation établie, il faut en effet revoir cette dernière, mais aussi conséquemment les exemples déjà catégorisés. D'un autre côté, la formalisation en cours des UST en Motifs Temporels Paramétrés, MTP, (Bootz \& Hautbois, 2007), permet déjà d'en fournir les propriétés définitoires et classificatoires et les ajustements à opérer. Enfin, la réflexion théorique sur le traitement cognitif de la dimension temporelle du flux sonore peut aider aussi à mieux définir les USTs. On peut envisager (Tijus, Poitrenaud, Bellissens, Heni \& Frémiot, 2004) que les données temporelles musicales soient codées en termes de mouvement spatial et selon un processus de schématisation spatiale. Dans une perspective biosémiotique qui postule la nécessité de prendre en compte le corps humain et son interaction avec l'environnement dans toute théorie de la signification (Varéla, Thompson \& Rosch, 1993), on peut aussi (Timsit-Berthier, 2004, 2007) mettre en correspondance les USTs aux familles de mouvements fonctionnels définies par Buytendijk (1957).

Quoi qu'il en soit, on ne mesure peut être pas suffisamment l'apport que constituerait pour l'étude des données temporelles l'équivalent de la géométrie pour les données spatiales. La géométrie définit un petit nombre, - de l'ordre d'une quinzaine - de figures caractéristiques. Ces figures planes géométriques ont comme constituants des points et des segments, droites et courbes, qui servent à les caractériser, à les identifier, à les catégoriser, et en cela à les différencier. On dispose ainsi pour l'espace 2D d'un répertoire constitué d'un petit ensemble de figures qui sont représentatives, abstraites, et opérationnalisables. Les USTs, comme figures sonomé-triques, présentent les caractéristiques de représentation, d'abstraction et d'opéra-tionnalisation pour les données temporelles et définissent 19 figures temporelles caractéristiques. 


\section{REMERCIEMENTS}

Ces travaux ont été financés en partie par l'Action Concertée Incitative "Terrains, Techniques, Théories, Travail Interdisciplinaires en Sciences Humaines et Sociales " du Ministère délégué à la Recherche et aux Nouvelles Technologies et par le CNRS.

Adresse pour correspondance:

Aline Frey

Laboratoire Cognitions Humaine et Artificielle

CHArt, Université Paris 8

2 rue de la Liberté

93526 St Denis (France)

e-mail: aline.frey@cognition-usages.org 


\section{- RÉFÉRENCES}

Bigand, E. (2006). Musiciens et non-musiciens perçoivent-ils la musique différemment? Dans Le Cerveau Musicien - Neuropsychologie et psychologie cognitive de la perception musicale. Bruxelles: De Boeck \& Larcier.

Bootz, Ph. \& Hautbois, X. (2007). Times Measures in Documents: The model of « Motifs Temporels Paramétrés ». In R. Skare, N. Lund \& A. Vårheim (eds), A Document (Re) turn. Frankfurt am Main: Peter Lang, pp. 197-222.

Buytendijk, F.J. (1957). Attitude et mouvements. Étude fonctionnelle du mouvement humain. Bruges : Desclée de Brouwer.

Chi, M. T. H., Feltovich, P. J., \& Glaser, R. (1981). Categorization and representation of physics problems by experts and novices. Cognitive Science, 5, 121-52.

Chion, M. (1983). Guide des objets sonores: Pierre Schaeffer et la recherche musicale. Paris, Buchet/ Chastel.

Cooke, D. (1959). On musical inspiration. In The language of music, pp. 168-75. Oxford: Oxford University Press.

Dehaene, S., Izard, V., Pica, P.. \& Spelke, E. S. (2006). Core knowledge of geometry in an Amazonian indigene group. Science, 311, 381-84.

Delalande, F. (1996). Les Unités Sémiotiques Temporelles : Problématique et essai de définition, in Les Unités Sémiotiques Temporelles, éléments nouveaux d'analyse musicale, pp. 16-25. Marseille : MIM (diff. ESKA, Doc. Musurgia).

Delalande, F., Formosa, M., Frémiot, M., Gobin, P., Malbosc, P., Mandelbrojt, J. \& Pedler, E. (livre-CD audio, 1996). Les Unités Sémiotiques Temporelles - éléments nouveaux d'analyse musicale. Marseille: MIM/Documents Musurgia.

Favory, J. (2007). Les Unités Sémiotiques Temporelles. Mathématiques et sciences humaines, $N^{0} 178$, Été 2007, spécial Art, mathématiques, langage et émotion.

Favory, J., Formosa, M., Frémiot, M., Gobin, P., Malbosc, P., Mandelbrojt, J. \& Prod'homme, L. (CD-Rom, 2002). Les Unités sémiotiques temporelles. Nouvelles cléspour l'écoute. Marseille: MIM.

Francès, R. (1958). La perception de la musique. Paris. Paris : Vrin.

Frémiot, M. \& Formosa, M. (2005). Analyse en UST de l'œuvre de Costin Miereanu « Les Labyrinthes d'Adrien ». In M. Formosa \& E. Rix (eds). Vers une sémiotique générale du temps dans les arts, pp. 21-32. Paris : Delatour.

Frémiot, M. (1999). "De l'objet musical aux Unités Sémiotiques Temporelles » in Oü̈r, entendre, écouter, comprendre après Schaeffer. Paris : INA-GRM/Buchet-Chastel.

Höppner, F. (2003). Knowledge Discovery from Sequential Data. PhD thesis, Technical University Braunschweig, Germany.

Imberty, M. (1991). Comment l'interprète et l'auditeur organisent-ils la progression temporelle d'une œuvre musicale ? (Analyse, mémorisation et interprétation). Psychologica Belgica, 31, 173-95.

Kramer, J. (2004). Le temps musical. Musiques. In J.-J. Nattiez (dir.), Musiques — Une encyclopédie pour le XXI siècle, 2 Les savoirs musicaux, pp. 189-213. Arles : Actes Sud/Cité de la musique.

Luce, R.D. \& Green, D.M. (1974). Detection, discrimination, and recognition. Handbook of Perception, Vol. II, pp. 299-342. New York : Academic Press. 
Mantel, N. (1967). The detection of disease clustering and a generalized regression approach. Cancer Research, 27, 209-20.

McAdams, S. (1993). Thinking in Sound: The Cognitive Psychology of Human Audition. Oxford University Press.

Messiaen, O. (2000). Traité de rythme, de couleur et d'ornithologie. Tome I. Paris : Alphonse Leduc.

Meyer, L. B. (1953). Emotion and Meaning in music. Chicago University Press.

Meyer, L. B. (1973). Explaining Music. Berkeley, Ca. : California University Press.

Nosofsky, R. M. (1992). Similarity scaling and cognitive process models. Annual Review of Psychology, 43, 25-53.

Pachet, F., Ramalho, G., \& Carrive, J. (1996). Representing temporal musical objects and reasoning in the MusES system. Journal of New Music Research, 25, 252-75.

Peretz, I. (2006) The nature of music from a biological perspective. Cognition, 100, 1-32.

Poitrenaud, S., Richard, J.F. \& Tijus, C. (2005). Properties, categories and categorisation. Thinking and Reasoning, 11, 151-208.

Prod'homme, L. (2005). "Applications pédagogiques des UST : quelques expériences. ", in M. Formosa \& R. Rix. (eds). Vers une sémiotique générale du temps dans les arts, pp. 23944. Paris : Delatour.

Reicha, A. (1824). Traité de Haute Composition musicale. Paris : Zetter \& Cie.

Reti, R. (1951). The Thematic Process in Music. New York : Macmillan.

Rosch, E. (1973). On the internal structure of perceptual and semantic categories, In T.E. Moore (ed), Cognitive Development and the Acquisition of Language, New York : Academic Press.

Rosch, E. \& Mervis, C.B. (1975). Family resemblances: studies in the internal structure of categories. Cognitive Psychology, 7, 573-605.

Ruwet, N. (1987). Methods of Analysis in Musicology. Music Analysis, 6, 4-39.

Sattath, S., \& Tversky, A. (1977). Additive similarity trees. Psychometrika, 42, 319-45.

Schaeffer, P. (1966). Traité des Objets musicaux : essai interdisciplines. Paris : Seuil.

Schering, A. (1936). Johann Sebastian Bachs Leipziger. Leipzig : Kirchenmusik.

Solomon, G.E.A. (1990). Psychology of novice and expert wine talk. American Journal of Psychology, 103, 495-517.

Tijus, C. (1986). L'identification de lettres dans diverses conditions de présentation. L'Année Psychologique, 86, 403-28.

Tijus, C. (2004). Introduction à la psychologie cognitive. Paris : Armand Colin.

Tijus, C. \& Cordier, F. (2003). Psychologie de la connaissance des objets : catégories et Propriétés, tâches et domaines d'investigation. L'Année Psychologique, 103, 223-56.

Tijus, C., Poitrenaud, S., Bellissens, C., Heni, J. \& Frémiot, M. (2004). La spatialisation du temps musical : les Unités Sémiotiques Temporelles. Actes des $11^{e}$ Journées CNRS de Rochebrune: Rencontres Interdisciplinaires sur les systèmes complexes naturels et artificiels, "Le temps dans les systèmes complexes", pp. 341-35, Paris : ENST.

Timsit-Berthier, M. (2004). Les Unités Temporelles Sémiotiques : un nouvel outil d'analyse musicale. Description et approche biosémiotique. Actes des $11^{e}$ Journées CNRS de Rochebrune: Rencontres Interdisciplinaires sur les systèmes complexes naturels et artificiels, "Le temps dans les systèmes complexes", pp. 353-63, Paris : ENST. 
Timsit-Berthier, M. (2007). Approche "Biosémiotique " des Unités Sémiotiques Temporelles. Mathématiques et Sciences Humaines, 2, 57-62.

Townsend, J.T. (1971). Theoretical analysis of an alphabetical confusion matrix. Perception \& Psychophysics, 9, 40-50.

Varéla, F., Thompson, E., \& Rosch, E. (1993). L’inscription corporelle de l'esprit. Sciences cognitives et expérience humaine. Paris : Seuil. 
Pertinence cognitive des unités sémiotiques temporelles A. FREY, A. DAQUET, S. POITRENAUD, CH. TIJUS, M. FREMIOT, M. FORMOSA, L. PROD'HOMME, J. MANDELBROJT, M. TIMSIT-BERTHIER, PH. BOOTZ, X. HAUTBOIS, M. BESSON

\section{- Pertinencia cognitiva de las unidades semióticas temporales}

En la línea de los trabajos pioneros de Pierre Schaeffer, el laboratorio Música e Informática de Marsella (MIM) ha puesto en evidencia figuras temporales sonoras denominadas Unidades Semióticas Temporales (UST) que corresponden a segmentos musicales que poseen, per se, una significación temporal en razón a su organización morfológica y dinámica (Delalande, 1996).

En este artículo, aportamos la descripción de las UST y los resultados de dos experiencias realizadas con la presencia de músicos y no músicos utilizando una tarea de categorización libre para evaluar la pertinencia de las UST, como unidades cognitivas de la escucha del flujo sonoro. Los resultados muestran que los segmentos musicales relevantes de una misma UST son reagrupados de forma significativa y que no hay diferencias entre músicos y no músicos en la aprehensión de las UST. Estos resultados son discutidos desde la perspectiva de la formalización de las UST.

\section{- Pertinenza cognitiva delle unità semiotiche temporali}

Partendo dal percorso di ricerca tracciato da Pierre Schaeffer, il laboratorio Musique et Informatique de Marseille (MIM) ha evidenziato figure sonometriche denominate Unités Sémiotiques Temporelles (UST) corrispondenti a segmenti musicali che hanno un significato temporale intrinseco dovuto alla loro organizzazione morfologica e dinamica.

In questo articolo mettiamo in relazione la descrizione delle UST e i risultati di due esperimenti nei quali i partecipanti, musicisti e non musicisti, risolvono un compito di categorizzazione libera, al fine di valutare la pertinenza delle UST come unità cognitive dell'ascolto del flusso sonoro. I risultati mostrano che i segmenti musicali rilevanti di una stessa UST si raggruppano in modo significativo e che non c'è alcuna differenza tra musicisti e non musicisti nel trattamento delle UST.

\section{- Cognitive Relevance of Temporal Semiotic Units}

Following the pioneered work of Pierre Schaeffer, the laboratory Musique et Informatique de Marseille(MIM)highlightedsonometrictemporalfigureslabeled Unités Sémiotiques Temporelles (UST)thatcorrespondtomusicalsegmentswhich have intrinsically a temporal significance because of their morphological and dynamicorganization.

This article relates to the cognitive relevance of UST, as cognitive units for processing. CognitiverelevanceofUST wasevaluatedintwoexperimentswith musicians and non musicians as participants solving a free categorization task. ResultsshowthatmusicalsegmentsofasameUSTaregroupedtoasignificant degreeandthatthereisnodifferencebetweenmusiciansandnonmusicianswhile processing UST. These results are discussed from the point of view of the formalization of UST. 
- Die kognitive Relevanz zeitlicher semiotischer Einheiten

Vor dem Hintergrund der Arbeiten von Pierre Schaeffer untersucht das Labor "Musique et Informatique de Marseille" (MIM) zeitliche Klangfiguren, die Unités Sémiotiques Temporelles (UST) genannt werden. Diese Figuren korrespondieren mit den musikalischen Segmenten, die per se durch ihre morphologische und dynamische Organisation zeitliche Bedeutung besitzen. In diesem Artikel beschreiben wir die UST und die Ergebnisse von zwei Experimenten mit Musikern und Nichtmusikern, die eine freie Kategorisierungsaufgabe lösten. Dadurch sollte die Relevanz von UST als kognitiven Einheiten des hörbaren Klangverlaufs evaluiert werden. Die Ergebnisse zeigen, dass die musikalischen Segmente derselben UST sich auf signifikante Weise gruppieren lassen, und dass es keine Unterschiede zwischen Musikern und Nichtmusikern im Verständnis der UST gibt. Diese Ergebnisse werden hinsichtlich einer Formalisierung der UST diskutiert. 